\title{
Pulmonary Vein Dominance
}

National Cancer Institute

\section{Source}

National Cancer Institute. Pulmonary Vein Dominance. NCI Thesaurus. Code C127587.

The determination of whether blood flow in the pulmonary veins is greater during ventricular systole or diastole. 